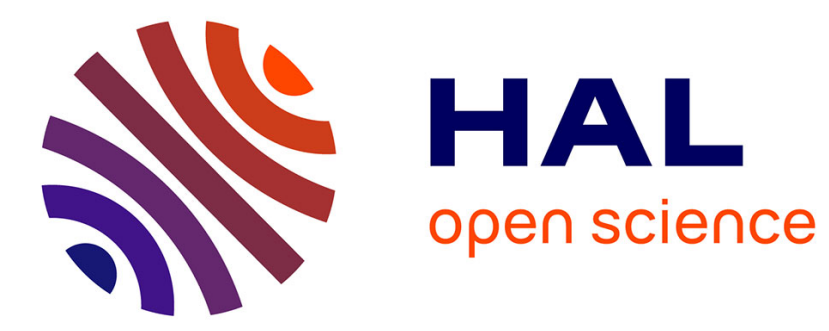

\title{
Le pouvoir local en débats. Pour une sociologie du rapport entre leadership et territoire
}

William Genieys, Andy Smith, François Baraize, Alain Faure, Emmanuel

Négrier

\section{- To cite this version:}

William Genieys, Andy Smith, François Baraize, Alain Faure, Emmanuel Négrier. Le pouvoir local en débats. Pour une sociologie du rapport entre leadership et territoire. Pôle Sud - Revue de science politique de l'Europe méridionale, 2000, Qui gouverne les viLLES „13, pp.103 - 119. 10.3406/pole.2000.1090 . hal-01393520

\section{HAL Id: hal-01393520 \\ https://hal.science/hal-01393520}

Submitted on 7 Nov 2016

HAL is a multi-disciplinary open access archive for the deposit and dissemination of scientific research documents, whether they are published or not. The documents may come from teaching and research institutions in France or abroad, or from public or private research centers.
L'archive ouverte pluridisciplinaire HAL, est destinée au dépôt et à la diffusion de documents scientifiques de niveau recherche, publiés ou non, émanant des établissements d'enseignement et de recherche français ou étrangers, des laboratoires publics ou privés.

\section{(ㅇ)(1) $\$$}

Distributed under a Creative Commons Attribution - NonCommercial - NoDerivatives $\mid 4.0$ 


\title{
Le pouvoir local en débats
}

\author{
Pour une sociologie du rapport \\ entre leadership et territoire
}

\author{
William Genieys, Andy Smith, François Baraize, \\ Alain Faure, Emmanuel Négrier ${ }^{1}$
}

CEPEL-CERAT-CERVL

\section{Résumé/Abstract}

Cet article propose de reconsidérer l'utilité de la notion de leadership à la lumière des changements qui affectent, depuis 20 ans, les cadres de l'action et du pouvoir territoriaux. Après une revue critique de la littérature sur la question, les auteurs envisagent le rapport entre leadership et territoire à la lumière de trois dimensions: la configuration territoriale, la construction politique et le rapport à l'identité territoriale du leadership. Ils fondent leurs analyses sur plusieurs études de terrain.

This article deals with the pertinence of the notion of leadership for improving the understanding of the changing patterns of territorial powerand action that have occured over the last twenty years. Having conducted areview of the existing approach to this question, the authors underline theimportance of a gap in this research: the relationship between leadershipand territory. As an antidote, they then propose a three dimensional analytical grid which simultaneously tackles: territorial configurations of power, the political construction of leadership and the role of territorial identiy. This proposition is illustrated with examples taken from a range of empirical research conducted by the authors themselves.

\section{$\approx$ \\ Mots-clés/Keywords}

Décentralisation, identité territoriale, leadership, notable, pouvoir local, territoire Decentralization, leadership, local power, notable, territorial identity, territory 
Les analystes de la décentralisation en France s'accordent à souligner que la réforme de 1982 a déstabilisé les piliers du pouvoir local. La remise en cause des rôles de l'État, l'engagement direct de la responsabilité des élus et la multiplication des actions publiques territoriales sont autant $d$ 'indices contradictoires et complexes sur les "génies invisibles du local" (Mabileau 1997). Le phénomène, fortement contingent du développement de nouveaux modes de légitimation des élus locaux, pose le problème de l'écart qui se creuse entre les outils de l'analyse des politiques publiques et ceux d'une sociologie de "la vie politique locale". Comment aborder de front le lien analytique qui sépare l'implication des élus dans les politiques publiques et leur activité de représentation politique? La connaissance sociologique sur les réseaux de décision et les "référentiels d'action publique" en présence se détache des questionnements portant sur le recrutement, la formation, l'action et l'évocation des élites politiques locales. Et la sociologie politique n'explique pas assez comment les élites agissent pour créer et maintenir leur position au sein des hiérarchies sociales complexes. L'établissement de ponts entre la sociologie de la représentation politique au local et l'analyse de l'action publique constitue un enjeu analytique stimulant. Pour préciser cette articulation, un des angles de lecture possibles concerne la notion de leadership politique. Dans les années soixante, la sociologie américaine du pouvoir local avait ouvert un chantier de recherche sur la nature décisionnelle, réputationnelle et positionnelle du pouvoir des leaders dans les collectivités territoriales (Freeman et alii., 1971). Mais ce courant d'analyse, qui s'intéressait principalement aux motivations des leaders, n'a reçu qu'un faible écho en France, notamment en raison de sa dimension psychologisante'. C'est bien l'analyse des rapports centre-périphérie et du "pouvoir périphérique ${ }^{3}$ " qui a prévalu pour rendre compte des modalités d'exercice du pouvoir local.

Néanmoins, depuis quelques années un virage s'opère quand le recours à la notion de leadership redevient une alternative constructive. En effet, certaines redéfinitions des approches mobilisant la notion permettent de dépasser la conception essentiellement interactionniste du pouvoir (Paige, 1977, 234), en questionnant directement les formes de légitimation des leaders 5 . Dans son ouvrage théorique sur le leader- 
ship, James MacGregor Burns souligne qu'à force de conceptualiser "la politique en tant que pouvoir", on en vient à sous-estimer "la question de la politique dans le pouvoir" $\left(1978,11^{6}\right)$. En s'émancipant d'une conception restrictive du pouvoir défini seulement comme un échange de ressources politiques "palpables", sa réflexion permet de placer au centre de l'observation la participation du leader à la production de nouvelles représentations du territoire, à l'affirmation des nouveaux acteurs politiques et aux mécanismes de recomposition de l'action publique.

Afin de comprendre ce glissement, il convient de revenir tout d'abord sur les apports et les limites de ces théories pour montrer ensuite en quoi un questionnement sur les nouvelles dynamiques du pouvoir local, notamment celle du rapport entre leadership et territoire, permet de concilier l'analyse de l'action publique locale avec celle de la représentation politique.

\section{La sociologie du pouvoir local en question (s)}

En France, les années quatre-vingt et quatre-vingt-dix sont le théâtre d'une importante recomposition du paysage institutionnel dans la mesure où de nombreux dispositifs d'intervention publique se "localisent" et se "territorialisent". Pour prendre la mesure de ces changements, le champ de la sociologie de la vie politique locale se déplace. Les travaux initiaux sur la structuration du politique (polity) et sur la compétition politique (politics) sont délaissés d'une part au profit de l'analyse des effets des politiques publiques locales (policies) et d'autre part à travers le développement d'une "sociologie critique" du pouvoir local.
Le notable comme pilier du système politique local

Des raisons socioculturelles propres à la France ont fait qu'en sociologie et en histoire, le notable est apparu comme la figure centrale du pouvoir local 7 . Pour les historiens, le concept de notable permet de dépasser le clivage entre noblesse et bourgeoisie. La tradition historique insiste particulièrement sur la dimension sociale du pouvoir notabiliaire en s'interrogeant sur les modes d'accès à la notabilité. Pour les tenants de l'histoire culturelle, le rapport noblesse/bourgeoisie est remis en cause avec l'apparition d'une "classe de notables" qui monopolise le pouvoir local dans la France post-révolutionnaire (Chaussinant-Nogaret, 1991, p. 281). De la même façon, les partisans de l'histoire sociale définissent les "grands notables" comme ceux qui, sous certains régimes politiques, cumulent un pouvoir économique, social et politique tant au niveau local qu'au niveau national (Tudesq, $1964^{8}$ ). Ainsi, le notable est défini, organiquement ou matériellement, à partir de la nature des ressources sociales (notoriété), économiques (richesse), politiques (élection) qu'il détient au sein de la configuration d'un régime politique particulier (Charle, 1997). Pour d'autres auteurs, les notables sont les élus locaux qui en entretenant des "guerres picrocholines" ont produit l'image d'une France conflictuelle (Birnbaum, 1998). Cette conception du notable est enrichie par le développement de la sociologie politique du pouvoir local qui met l'accent sur les modes mêmes d'exercice du pouvoir local.

La tradition sociologique reconnaît avec Max Weber le rôle politique particulier du notable dans le processus de développement de l'État moderne'. Toutefois, ces travaux tendent à distinguer au sein du personnel politique local le notable, celui qui vit pour la 
politique, du professionnel, par et de son activité politique ${ }^{10}$. A partir des années soixante, dans une filiation wéberienne centrée sur les articulations entre le pouvoir périphérique et l'administration étatique en France, les recherches du Centre de Sociologie des Organisations (CSO) reconsidèrent le phénomène notabiliaire ". Plus précisément, ces travaux privilégient l'analyse d'un système d'action où le notable entretient un rapport privilégié avec le pouvoir central et l'administration déconcentrée. Cette perspective met en exergue l'étude de la capacité des acteurs à se positionner au cœur des processus de décision. Le notable est celui qui occupe une position charnière entre le territoire et la bureaucratie de l'État français. Par conséquent dans cette approche fonctionnelle du pouvoir local, ce n'est plus uniquement le poids social du notable qui importe, mais les relations qu'il aura su tisser avec les représentants de l'administration locale, notamment le préfet (Worms, 1966). Ainsi, le "pouvoir périphérique" s'organise autour d'un système d'action où le notable accroît son pouvoir du fait d'une relation privilégiée à l'État tout en légitimant les décisions du préfet sur le territoire (Grémion, 1976). Une des limites de l'approche du CSO est qu'en faisant du rapport centre-périphérie son angle privilégié d'analyse, elle ne retient du local et des acteurs qui l'animent (les notables) que leur rôle de freins et d'entraves aux changements initiés par le centre. Cette conception du pouvoir local oppose donc implicitement des réformes institutionnelles et administratives voulues par l'État à un immobilisme de la périphérie. Pourtant, d'autres travaux montrent que la figure de l'homme politique local qui traite au coup par coup les problèmes individuels (le notable et son réseau) s'efface progressivement à partir des années 70 devant celle de l'elu "entrepreneur" (Lorrain, $1993^{12}$ ). Cet angle d'analyse est conforté par les recherches qui insistent sur les modifications substantielles des ressources du notable apparues avec la décentralisation (Rondin, 1985; Alliès, 1991).

En résumé, la sociologie du notable peut schématiquement être discutée et enrichie sur quatre points. Tout d'abord, la variable partisane, volontairement laissée de côté, mérite sans doute plus d'attention au moment où l'on assiste à un accroissement du rôle "localise" des partis politiques ${ }^{13}$ et de leur fonction de médiation et de redistribution des ressources politiques. Ensuite, il faut intégrer le fait que les lois de décentralisation réaffirment la place des élections locales comme enjeux politiques structurants du cycle électoral français (Gaxie, Lehingue, 1984; Gaxie 1997), tant pour les compétitions électorales municipales que départementales et régionales. Troisièmement, l'analyse des politiques publiques locales montre que le modèle de la régulation croisée est fortement perturbé par la multiplication des centres de décision et la territorialisation de l'action publique. Ainsi, le modèle "d'institutionnalisation de l'action collective" permet-il de rendre intelligible un pouvoir plus polycentrique que périphérique (Duran, Thœnig, 1996). Enfin, même si la rémunération de l'élu n'est pas forcément sa rétribution principale, le "métier d'élu local" (Fontaine Le Bart 1995) connaît des évolutions considérables. À côté des modes opératoires traditionnels (notamment hors agglomérations), d'autres pratiques se font jour (Faure, 1992, 1997). Elles appellent une sociologie critique du pouvoir local qui doit se recentrer sur les dimensions politiques et sociales de l'activité politique mais aussi sur l'analyse de l'implication de l'élu dans l'action publique locale. 


\section{Les figures du professionnel et de l'éligible}

Plusieurs courants de recherches développent aujourd'hui une approche critique du pouvoir périphérique en insistant sur le rôle des élus (au sens large) dans la vie politique locale. Dès lors, les politistes vont s'interroger plus précisément sur les effets de la professionnalisation de la vie politique locale tandis que des anthropologues soulignent les effets de la transformation des modes de légitimation du pouvoir notabiliaire. L'analyse de la constitution du leadership politique de Jacques Chaban-Delmas fait à cet égard figure de travail pionnier (Lagroye, 1973). Cette recherche montre comment le maire de Bordeaux développe, exploite et renforce les logiques sociales et politiques de "la société bordelaise" pour asseoir territorialement son pouvoir. L'auteur met en évidence la multiplicité des ressources qui participent à la construction d'un leadership politique sur un territoire donné. Dans ce cas particulier, le pouvoir du leader est lié aux pratiques politiques qui lui permettent de jouer de la cohésion sociale du "milieu bordelais". C'est dans le prolongement de ces interrogations que se développe l'analyse du pouvoir local comme espace politique permettant de saisir les logiques de la professionnalisation des activités politiques. Les travaux sur les professionnels de la politique mettent l'accent sur le temps consacré par l'élu local à l'activité politique, le rôle du salaire ou encore l'influence croissante des partis politiques (Gaxie, 1973). Ainsi, une étude sur le comportement des maires urbains montre que ces élus ont en commun la mise en avant d'un savoir-faire politique particulier (Garraud, 1989). Dans cette perspective, la constitution d'intérêts propres à l'activité politique devient la caractéristique principale de la professionnalisa- tion. Toutefois, on peut relever ici les ambiguïtés économicistes des catégories d'analyse employées (profession, entreprise, monopole...) et la tendance à présenter uniquement l'activité politique comme le résultat de choix rationnel. Le terme de métier politique permet à cet égard d'ouvrir des pistes de recherche où l'action politique ne résulte pas seulement de l'agrégation volontaire d'intérêts particuliers. Pour Philippe Garraud $\left(1994^{14}\right)$, la "marque du métier politique" se caractérise par la conquête et l'exercice du pouvoir, l'élu local étant un intermédiaire (un médiateur, un passeur) établissant une communication entre différents segments ou réseaux de la société locale (Garraud, 1994, p. 40). Une autre approche du métier politique consiste à modérer l'importance des pratiques partisanes pour souligner de façon plus globale "l'inscription sociale du politique ${ }^{15}$ ". Toutefois cette sociologie "critique" du pouvoir local tend à laisser de côté la question de la mobilisation des ressources de l'action publique et l'articulation, souvent décisive, qui s'opère dans les relations entre les élus locaux et les fonctionnaires territoriaux (Gaudin, 1999).

L'anthropologie politique ouvre aussi de nouvelles pistes de recherche sur le pouvoir local. Contrairement aux politistes, les anthropologues mettent au centre de leurs préoccupations le territoire, ses rituels et ses symboles. Plus précisément, ils insistent sur l'imbrication du politique et des autres dimensions du social (Abélès, 1997). L'analyse du pouvoir politique local s'intéresse ici aux différents modes de légitimation politique des élus dans une optique résolument noninstitutionnelle, privilégiant le rapport de l'élu au territoire en lieu et place de la métaphore désincarnée des allégeances centre-périphérie. C'est sur le territoire que s'affrontent les identités, se découpent les espaces et se développent les pratiques et les représentations qui 
façonnent la sphère du politique. Dans son étude de la configuration politique locale du département de l'Yonne en période "postdécentralisation", Abélès étudie l'affirmation d'un pouvoir "concentrique" autour du Président du Conseil général (1989, chapitre IV). Le concentrisme n'est pas seulement une affaire de réseaux d'hommes politiques, d'agents de l'État et des partenaires sociaux. Il révèle comment les élus sont confrontés au niveau du territoire à une double contrainte avec les instances externes et l'ancrage sur un territoire ${ }^{16}$. En filigrane, cette analyse reconnaît que le leadership politique émerge sur le territoire, non seulement parce que l'élu local peut afficher le soutien des socio-professionnels ou s'imposer comme un professionnel des dossiers et de la décision publique, mais aussi parce que son élection reflète une façon de travailler le territoire en tant qu'eligible. Partant d'une perspective d'anthropologie historique, Yves Pourcher développe cette deuxième dimension dans son étude de la dévolution du pouvoir en Lozère (1987), en montrant le processus d'identification continu des notables au territoire. C'est à travers les stratégies de reproduction, d'alliance et d'héritage que se dessinent les mécanismes sociopolitiques par lesquels ces élus s'accaparent les logiques par- tisanes et imposent leur légitimité sur un territoire donné.

Les approches critiques de la vie politique locale ont donc permis de réintroduire certaines particularités propres à l'activité politique de l'élu local que l'approche fonctionnaliste du pouvoir notabiliaire a sous-estimé. Néanmoins, cette lecture ne nous informe pas suffisamment sur la façon dont les élus locaux utilisent les ressources de l'action publique pour accroître leur légitimité politique. Il est possible de schématiser sous forme de tableau l'apport de ces différents courants (en termes de notables, de métier et d'éligibilité) à l'analyse du pouvoir local.

Ce tableau récapitulatif montre deux lignes de partage intéressantes. La première concerne la place centrale ou secondaire de l'action publique dans les différentes grilles d'analyse des élites locales. La seconde oppose les partisans d'une prise en compte de la mobilisation symbolique du territoire à ceux qui préferent s'en tenir à l'étude de ses ressources concrètes. Cette perspective à quatre entrées permet également de problématiser l'opposition idéologique du clivage centre-périphérie entre tradition et innovation. Autrement dit, il convient de recentrer l'analyse du pouvoir local sur une sociologie des rapports entre leadership et territoire.

Les variables dPanalyse du pouvoir local

\begin{tabular}{l|c|c|c|c}
\hline variables/courants & $\begin{array}{c}\text { Trajectoires } \\
\text { politiques }\end{array}$ & $\begin{array}{c}\text { Action } \\
\text { publique }\end{array}$ & $\begin{array}{c}\text { Changement } \\
\text { institutionnel }\end{array}$ & $\begin{array}{c}\text { Identités } \\
\text { temitoriales }\end{array}$ \\
\hline Notable (CSO) & O & E & E & C \\
Professionnel/métier & E & 0 & 0 & C \\
L'éligible & E & 0 & 0 & E \\
\hline
\end{tabular}

$E=$ dimension essentielle $; O=$ dimension occultée $; C=$ dimension critiquée 
La capacité des élus à mobiliser des ressources politiques ne se limite pas à des logiques d'organisation et d'échange politique mais plutôt à un processus plus global où le leader motive ses followers en se référant au territoire (Pôle Such, 1997). Il s'agit alors de montrer en quoi, confrontés à la réalité de la décentralisation, les élus locaux sont amenés à développer certaines pratiques managériales qui modifient leur appréhension du pouvoir périphérique. La prise en compte du rapport leadership/territoire permet de restituer la particularité des trajectoires politiques des leaders. Au gré de chaque configuration politique et territoriale, ces derniers doivent affirmer leur capacité à transformer le territoire tout en acceptant la portée des réseaux et des intérêts en présence. Bref, pour affirmer leur leadership politique ces élus doivent simultanément intégrer dans leur stratégie politique les dynamiques sociales du territoire mais aussi les opportunités que leur offrent les changements institutionnels.

\section{Les trois dynamiques du rapport entre leadership et territoire}

Comment s'engager dans une analyse du pouvoir local qui dépasse les perspectives aterritoriales en s'enrichissant de la sociologie de chaque territoire concerné? Cette exigence et cette quête, qui impliquent en même temps que les réflexions comparatives dépassent le constat des singularités historiques et sociopolitiques propres à chaque cas d'étude, nous incitent à adopter un modèle d'analyse basé sur les "configurations" de l'action politique locale. Cette posture vise à établir et à maintenir une distinction analytique entre les modes d'intervention d'un élu local (qui peuvent se ressembler, voire converger) et les configurations d'émergence du leadership politique (qui seront toujours distinctes et propres à un territoire). Le terme de mode d'intervention recouvre les pratiques politiques qui sont individualisables. Faire un discours républicain le 14 juillet, centraliser la machine interne d'un parti, "monter à Bruxelles" pour s'assurer d'une aide communautaire, constituent des modes d'action politique susceptibles d'être développés par un élu local. Dans une perspective de management public ciblée sur l'efficacité ou l'efficience, ces interventions peuvent être étudiées et comparées en profondeur. Mais en soi cela n'informe guère sur le sens social de la pratique en question, ni du rapport entre le leader, sa légitimité et son territoire.

Nous suggérons ici que ce rapport s'articule sur trois dimensions. La configuration territoriale permet d'envisager l'enracinement politique et social des contraintes et des opportunités de leadership. La construction politique centre l'étude sur les trajectoires personnelles, dans leurs relations aux entourages, équipes et followers. L'identité territoriale se rapporte aux registres de présentation et de représentation que portent les leaders. Travailler sur l'imaginaire collectif, et son rapport à l'espace politique, c'est aussi, loin des métaphores bucoliques, appréhender autrement l'action publique territoriale.

\section{Intervenir sur quoi?}

\section{Les configurations territoriales comme enjeux d'action publique}

Le concept de configuration, tel qu'il est formalisé par Norbert Elias (1991 ${ }^{17}$ ), peut se révéler utile pour restituer la polysémie du territoire, ou plutôt des territoires d'action dans lesquels se meut l'élu local. Cet outil peut être fertile pour analyser les différentes conceptions du territoire, de l'électif (la circonscription électorale) au gestionnaire (la 
mise en cuvre de l'action publique locale) en passant par l'identitaire (la culture locale). Ces différents territoires constituent autant de configurations territoriales auxquelles se trouvent confrontés tous les élus locaux en quête de leadership. Un élu local peut tout à la fois agir sur son territoire d'élection (sa circonscription électorale) et mettre en cuvre des politiques publiques fondées sur la valorisation des identités locales. Mais il peut aussi intervenir au sein d'une seule de ces configurations territoriales. $\bar{A}$ une époque de recomposition accélérée du pouvoir local (décentralisation, déconcentration, "processus autonomique", intégration européenne, privatisation...), il nous paraît nécessaire d'établir une grille d'analyse permettant de croiser un questionnement sur les rapports instables entre la représentation politique, le territoire et le changement institutionnel, c'est-à-dire entre les trois variables que sont le leadership politique, les configurations territoriales et le changement institutionnel.

Avec l'essor de l'analyse des politiques publiques locales, le territoire s'est progressivement imposé comme une variable d'analyse potentiellement structurante. Dans cette perspective, la sociologie des organisations et la sociologie des réseaux d'action publique soulignent l'importance des formes d'interaction sectorielles et/ou territoriales (Friedberg, 1993 ; Cole, John, 1995). Cependant, ces travaux sur la territorialisation de l'action publique laissent de côté la question de l'implication des élus locaux dans l'action publique au profit d'une réflexion sur la "capacité politique des territoires" (Balme et alii., 1994). Pour d'autres chercheurs, la "négociation territoriale" devient un passage obligé du processus de légitimation de l'action publique locale (Gaudin, 1995). Aujourd'hui encore, la plupart des travaux de synthèse sur la sociologie de l'action publique locale lais- sent de côté la question de l'articulation entre la formation de leadership locaux et la mobilisation des ressources de l'action publique sur le territoire (Duran, Thœnig, 1996). De même, l'implication des élus locaux dans les politiques publiques est souvent perçue comme une dimension nouvelle du clientélisme politique (Briquet, 1997). En résumé, l'analyse des politiques locales fait l'économie d'une réflexion sur les effets matériels et symboliques de l'action publique sur la trajectoire des élus locaux. Pourtant, le territoire constitue un lieu privilégié pour observer les effets de "légitimation croisée" qui s'établissent autour de l'action d'un prétendant au leadership politique. Des lors, il convient d'analyser comment un élu local prend en compte les représentations sociales du problème à résoudre, présentes sur son territoire, et essaie d'y répondre en participant à la formulation de nouvelles politiques publiques. Cette question déborde aujourd'hui largement le seul cercle des élus. Elle vise, par exemple, les représentants de l'État sur le territoire. L'une des leçons de la décentralisation est en effet que les ressorts et la mise en œuvre concrète de ce pouvoir de représenter varient fortement d'un territoire à l'autre. D'une certaine manière, la question de savoir comment et jusqu'où être suivi s'est emparée de la préfecture, et ce sur les terrains mêmes de l'action politique locale. Marc Smyrl avait, l'un des premiers, mis en évidence les nettes différences de comportement des préfets dans une même responsabilité de mise en ouvre régionale des fonds structurels européens (Smyrl 1995). En analysant le rôle des préfets dans les projets d'agglomération, nous avons pu mettre en évidence trois "rôles" (l'assureur, l'entrepreneur et l'épargnant) qui correspondent à trois configurations territoriales distinctes (Baraize, Négrier 2000b). Cet exemple montre bien que la notion de leadership, loin 
de n'être que l'application d'une vision du monde politique comme "échange de ressources politiques palpables" (cf. supra), entretient avec ces dernières un rapport dialectique: à une configuration donnée correspond, pour le leader préfectoral, tel type de ressource pertinente afin d'étendre ses chances d'être suivi. Habitués, dans l'ordre républicain, à compter sur le même éventail de dispositifs publics, les préfets doivent aujourd'hui apprendre de leur territoire ceux sur lesquels leur pouvoir de représentation pourra s'incarner.

En prenant en compte les idées dans l'action politique comme des objets de négociation au sein des "arènes" et des "forums" des politiques publiques (Jobert, 1996), il semble possible d'étudier les processus de définition des problèmes publics à l'aune du rapport qui s'établit entre les politiques publiques et les identités partisanes, professionnelles et territoriales en $\mathrm{jeu}^{18}$. Mais l'application de ce schéma d'interprétation sous-estime souvent la spécificité sociologique et territoriale des acteurs étudiés. Le risque est alors grand de recentrer l'analyse sur les structures institutionnelles, en analysant alors des mouvements d'idées détachés de leurs émetteurs. Pour éviter ce biais, il semble important d'observer la manière dont s'établit et s'entretient la confiance entre les élus locaux mobilisant les ressources de l'action publique et les administrés, dans la mesure où le territoire en constitue le lieu de résonance. De manière générale, tout leader politique se trouve confronté au dilemme posé par les contradictions entre des logiques propres à l'action publique et celles de la demande sociétale. Agissant comme un entrepreneur potentiel des politiques publiques, l'élu doit parallèlement se prononcer sur les controverses générales liées à la mise en ouvre de politiques sectorielles tout en prenant en compte les spécificités du territoire qu'il représente ${ }^{19}$. On peut voir une bonne illustration de cette question dans l'étude du rôle des présidents de région dans la mise en ouvre des politiques régionales (Le Pape, Smith, 1999). On a notamment pu constater que malgré les différences entre les trajectoires de leaders régionaux, la relation à la configuration territoriale de l'action publique exprimait des relations structurantes d'allégeance et de clientélisme. La négociation du contrat de plan avec l'Etat et l'affirmation des politiques européennes font que cet échelon politique connaît un fort développement et participe activement à la difficile genèse d'une élite politique régionale (Genieys, 1997c). Les résultats des élections régionales de mars 1998 ont en quelque sorte confirmé cette tendance à l'autonomisation du leadership politique des présidents sortants, et l'échec des étatsmajors partisans ${ }^{20}$.

\section{Comment intervenir? Trajectoires et entourages}

De manière générale, la notion de leadership politique caractérise une catégorie d'acteurs qui, tout en se référant à la défense de "l'intérêt général", s'attachent à établir et à étendre leur autonomie politique. Formellement, ce phénomène se traduit par une façon de constituer et de mobiliser une équipe ou un entourage politique qui entre en relation avec un parti politique et qui met en scène le pouvoir. Toutefois, l'éligibilité des leaders n'est pas limitée à la filiation partisane (tendance de l'approche des professionnels de la politique) et à l'hérédité politique (tendance des anthropologues). Les leaders potentiels et confirmés travaillent leur entourage politique. Les travaux de Francis G. Bailey sur l'émergence du leadership dans la société indienne montrent que le leader est celui qui monopolise et qui gère les ressources dans une 
situation politique particulière (Bailey 1971, pp. 49-73). La capacité entrepreneuriale de redistribution du leader révèle notamment un entourage plus ou moins performant selon que ce dernier s'appuie sur des "mercenaires" ou sur des "fidèles". Cette distinction nous paraît très importante à l'heure où les élus locaux possèdent de plus en plus de responsabilités et doivent intervenir régulièrement dans les politiques publiques ${ }^{21}$. Toutefois cette proposition doit être enrichie par une entrée qui permet de mieux comprendre des actes de représentation, au sens large, où se superposent des logiques d'invention du territoire et "le défi de l'authenticité" dont parle Robert Starratt dans sa sociologie des leaders (1993, 48-51). Sa proposition visant à distinguer le leadership fonctionnel du leadership substantif évoque deux formes d'intervention politique consubstantielles esquissant une conciliation entre la sociologie du pouvoir local et l'analyse des rapports entre leadership et territoire.

Il faut aussi prendre en compte la manière dont le leader conçoit et travaille sa relation à l'espace et au temps. Dans cette perspective, la configuration du territoire n'est pas seulement considérée comme un lieu d'action publique où l'élu local interagit principalement avec les acteurs des instances publiques, parapubliques et privées. Elle est également une source de référents symboliques que le leader politique peut, et dans un sens doit "instrumentaliser", pour s'imposer comme un représentant politique légitime auprès de ces mandants administrés. La configuration territoriale revêt aussi une dimension centrale dans la constitution d'un lien privilégié avec ses propres soutien $s^{22}$. Toutefois, si l'élu local peut jouer de ces différentes dimensions du territoire, il ne peut pas le faire n'importe comment et surtout n'importe quand. La configuration territoriale n'est pas une simple pièce ou un lieu de ressources, elle est aussi, et d'abord, une composante-clé du lien social ${ }^{23}$. Par conséquent, un acteur politique doit non seulement tenter d'incarner les représentations traditionnelles du territoire, mais il doit aussi en promouvoir sa modernisation. Ces deux obligations correspondent à ce que Robert Starratt caractérise comme le leadership substantif, autour de pratiques qui "tendent à souligner le sens, les objectifs englobants et l'identité qui motivent et qui guident les personnes d'une société ou d'une organisation" $(1993,4)$. Cette dimension substantive, qui complète l'approche instrumentale, permet d'intégrer les représentations territoriales en tant que facteur de légitimation de l'activité politique des élus.

Ce dernier point prend tout son sens dans la période contemporaine de changement institutionnel accéléré ${ }^{24}$. Les situations d'affaiblissement des institutions, tel que le changement de régime, sont des moments privilégiés pour l'affirmation de la capacité d'innovation des leaders (Genieys, 1997a ${ }^{25}$ ). Certes, d'un point de vue formel, le régime politique français n'a pas connu un tel changement depuis l'instauration de la Ve République. Cependant, au cours des années quatre-vingt, le paysage institutionnel du pouvoir local a connu une transformation conséquente. Le réagencement des échelons politico-administratifs modifie les ressources mais aussi le cadre territorial d'intervention des acteurs politiques locaux. L'émergence d'institutions nouvelles comme le Conseil régional ou encore le développement de l'intercommunalité constituent autant d'espaces politiques où la prise en compte des rapports leadership/territoire permet de comprendre comment les élus locaux se construisent de nouvelles formes de légitimité (Faure, 2000). La mise en ouvre des Communautés d'Agglomération constitue un lieu privilégié pour analyser au concret le 
rapport leadership/territoire. Bien des ingrédients de cette fluidité conjoncturelle s'y retrouvent en effet: nouveauté de l'espace d'action, émergence de recettes inédites, incertitudes nombreuses sur les principes et niveaux de coopération... Dans ce cadre, on voit bien comment les élus locaux jouent des décalages entre leur territoire d'élection, qui pour l'instant ne change pas, et leur territoire d'action publique, qui se trouve substantiellement modifié (Baraize, Négrier, 2000a). Dès lors, outre l'importance des enjeux financiers, c'est bien souvent la dimension symbolique, notamment la capacité à incarner ce nouveau territoire, à lui donner une chair suffisante, qui justifie que les "followers" suivent le leader.

\section{Intervenir au nom de quel territoire? Le changement institutionnel et les dynamiques identitaires}

L'analyse du rapport leadership/territoire ouvre aussi la voie d'une réflexion sur les dynamiques politiques qui dépassent le strict cadre des institutions politiques traditionnelles. Ainsi, il est clair que l'on retrouve l'expression de ces dynamiques dans une situation institutionnelle où la légitimité des territoires est susceptible d'être contestée. Le territoire d'un leader politique revêt une forme duale. Il apparaît d'une part comme un espace d'interactions où les acteurs sont engagés dans une lutte politique pour déterminer leurs droits d'intervention, leurs ressources et donc leur degré d'autonomie institutionnelle (Le Galès, 1995). Il révèle d'autre part un espace identitaire, le territoire étant un lieu d'appartenances recomposé autour des objets de la vie quotidienne des habitants qui y trouvent une résonance et un ancrage (Taylor, 1989). Dans son travail de représentation politique, tout élu politique est en permanence confronté à ces deux conceptions dyna- miques du territoire. Cependant, à quelques exceptions près comme la Catalogne ou l'Écosse, ces conceptions sont toujours en plus ou moins grand décalage l'une par rapport à l'autre. Ce décalage appelle deux séries de commentaires sur la formation du lien social et sur les conceptions du territoire.

D'une part, il semble indéniable que "l'illusion identitaire" relève du développement de stratégies de certaines élites en quête de pouvoir (Bayart, 1996). En ce sens, la mobilisation des identités territoriales influe fortement sur les modes de construction de la représentation politique au sein de certaines périphéries $^{26}$. En Espagne par exemple, les leaders politiques de certaines régions mobilisent explicitement les identités territoriales pour faire aboutir des projers de réforme de l'État (Genieys, 1997b). Même s'il faut les distinguer des politiques de "marketing territorial" (Le Bart, 1992), ces stratégies montrent en France l'implication de certains élus locaux dans des politiques intercommunales de développement culturel (Faure, Smith, 1998). D'autre part, les différentes conceptions du territoire de l'action politique ne correspondent plus, bien souvent, au découpage institutionnel traditionnel. Paradoxalement, on assiste à l'émergence de territoires dotés d'un fort potentiel d'action publique où la légitimation par l'élection directe est faible. Les nouvelles structures intercommunales et l'institution régionale relèvent de ce cas de figure en France. Le développement de politiques interterritoriales voire transfrontalières confirme ce phénomène. En agissant dans le cadre de politiques interterritoriales, les leaders politiques définissent en permanence les frontières de leur zone d'action (Jessop, 1996). C'est en partie à travers la définition d'un "imaginaire spatial" qu'ils construisent leur légitimité politique. L'affirmation du leadership politique de l'élu local provient alors 
de la capacité qu'a ce dernier à jouer sur les différentes configurations territoriales, notamment celle des identités locales.

Dans les années 80 , cette dimension de l'imaginaire spatial a eu une résonance particulière s'agissant des Régions. Dans leur travail de construction politique d'un territoire, la manipulation de l'identité a fait figure $\mathrm{d}$ 'instrument du pauvre. Les élus régionaux étaient d'autant plus enclins à affirmer leur "identité territoriale", historique, culturelle...,etc, qu'ils étaient politiquement des nains, à côté des élus urbains ou départementaux, des rouages étatiques ou de leurs voisins européens. C'est oublier que cette construction imaginaire a eu parfois des effets tout à fait significatifs en termes de politique publique. À titre d'exemple, le cheminement de Jacques Blanc, président du Conseil Régional du Languedoc-Roussillon, est caractéristique. Sa "découverte" de la manière dont Jordi Pujol, président de la Communauté Autonome de Catalogne, utilisait la ressource historique et culturelle pour accroître son emprise sur son territoire a eu un effet décisif sur sa propre mise en œuvre d'une politique "occitane" en Languedoc-Roussillon. Ce mimétisme imaginaire était loin d'être évident : l'occitanisme de gauche ne lui était pas acquis, son alliance avec le Front National lui imposait une contrainte de poids, les milieux catalanophones et occitanophones étaient sujets à des controverses internes... Pourtant, dix ans plus tard, la politique languedocienne en matière de langue régionale était reconnue comme l'un de ses principaux fleurons. Elle avait permis, au passage, au président de l'exécutif d'étendre son leadership dans des espaces sociaux traditionnellement acquis à la gauche (Négrier 1998).

L'étude de la mise en œuvre de certaines politiques européennes ${ }^{27}$ permet d'observer les nouvelles opportunités de politique publique qui mobilisent des élus locaux souhaitant affirmer leur leadership. La question de la construction des identités constitue parfois pour ces territoires un enjeu prépondérant dans la consolidation du pouvoir de l'élu local. Une analyse comparée des effets de ces changements sur la formation d'un leadership politique dans le "Pays cathare" audois et dans les "Baronnies" drômoises permet par exemple de souligner la différenciation des logiques de mobilisation du territoire (Genieys, Smith, 1998). La mise en œuvre d'un développement économique peut donner à la valorisation des ressources identitaires du territoire une place déterminante, comme le montre la trajectoire politique de l'acteur politique qui impose son leadership dans le projet Pays cathare (Genieys, 1997b et 1999). Dans un autre contexte, les élus locaux adoptent une rhétorique culturelle qui ne débouche pas sur la mobilisation des identités du territoire. L'exercice comparatif montre que c'est principalement dans des situations où le changement affecte les institutions et les politiques que certains acteurs se montrent capables de développer des nouvelles formes de légitimation. Or, force est de reconnaître qu'actuellement la mobilisation d'imaginaires territoriaux par les leaders politiques reste une pratique peu étudiée dans les sciences sociales, ou exclusivement limitée aux territoires ruraux. Incontestablement, le "frémissement intercommunal" qui traverse le système local français met aujourd'hui les élus-majors à l'épreuve d'un subtil travail de redéfinition des valeurs au sein des communautés d'agglomérations, des communautés de communes et des "pays". C'est donc à partir de l'analyse des interactions multiples, matérielles et imaginaires, qu'entretiennent les élus locaux avec le territoire concret, que l'on peut mieux comprendre les processus de construction du leadership politique. 
Les spécialistes français du pouvoir local insistent souvent sur l'idée qu'en mettant l'accent sur les dynamiques d'action publique, la science politique risque de perdre de vue les fondamentales qui font l'essence du politique. La notion de leadership apparaît ainsi rarement saisie par les politistes pour ses vertus heuristiques, que ce soit par peur des vieilles lunes du behaviorisme ou des enfermements biographiques. Pourtant, il nous semble que la réflexion sur le leadership constitue une belle occasion pour (ré) concilier les sociologies de l'action et ceux du pouvoir territorial. Les travaux d'analyse de la vie politique locale ne prennent pas toujours la mesure de cette dimension, comme si, d'une part, la légitimité de l'espace territorial d'action de chaque élu n'était pas discutable et d'autre part l'instrumentalisation du territoire au travers de l'action publique locale ne suscitait guère de controverses. Or, les dynamiques du pouvoir mettent quotidiennement en question de telles certitudes, dans une mise en scène politique où se côtoient des trajectoires politiques, des ressources, des changements institutionnels et des identités locales. Autant de thèmes interdépendants que l'approche par le leadership permet peut-être de mieux relier et de mieux comprendre.

\section{Notes}

1. Ce texte a pour origine une communication de W. Genieys et $A$. Smith au congrès de l'Association Française de Science Politique à Rennes en septembre 1999. Il a ensuite fait l'objet d'un débat dans le cadre d'une Aide à Projet Nouveau du CNRS puis d'une nouvelle formulation a laquelle ont contribué F. Baraize, A. Faure et E. Négrier.

2. Dès les années cinquante, François Bourricaud présentait l'état de la sociologie du leadership telle qu'elle ressort de l'étude des groupes restreints dans les travaux anglo-saxons. Il avance l'hypothèse que ce type d'analyse "est susceptible d'être généralisé et appliqué à l'étude des phénomènes politiques", (Bourricaud, 1953, p. 446).

3. Il s'agit des travaux pionniers du Centre de Sociologie des Organisations sur l'adaptation du pouvoir local aux réformes de l'administration française, (Crozier, Thoenig, 1975; Grémion, 1976).

4. Cette posture analytique est sous-jacente à l'approche de la communauté politique (community power) avancée par Robert Dahl dans son imposante monographie sur "Qui gouverne à New Haven?" (1961). Selon ce sociologue, un leader politique, notamment un maire urbain, peut jouer le rôle de catalyseur de la communauté politique, rôle qui dépend surtout de sa capacité à mobiliser des ressources interpersonnelles. Plus explicitement, pour Dahl, le leadership politique se mesure à travers la capacité qu'ont ces acteurs à hiérarchiser les ressources d'un territoire en établissant des compromis au sein des coalitions gagnantes (winning coalitions) avec les acteurs sociaux locaux avant et après les élections municipales.

5. Robert Tucker rappelle que "l'approche en termes de rapport de forces (the power approach) ne nous éclaire ni sur ce que font les leaders, ni sur ce qu'on attend qu'ils fassent en tant que leader" (Tucker, 1995, 8).

6. Ce courant de recherche participe à ce qu'Aaron Wildavsky qualifie "d'acte de magie où l'on fait disparaître le leadership" où "toutes les voies trackes pour étudier le leadership finissent par dissoudre l'objet même de recherche", cf. l'interview publice dans The New York Times Review of Books, 27 april 1980, p. 12.

7. Rappelons que la notion de notable est une catégorie d'analyse franco-française. On ne trouve aucune rubrique dans The Blackwell Encyclopedia of Political Science (1991). De même, il semble que l'emploi du terme de notable dans la version française de Qui gouverne? relève d'un effet de traduction dans la mesure où Robert Dahl n'utilise dans la version originale que le seul terme de notability. 
8. Pour André Jean Tudesq "la domination des notables correspond a la fois à la confusion des trois pouvoirs économique, social et politique et à la concordance entre le pouvoir exercé sur le plan local et le pouvoir dominateur sur le plan national" (1964, vol II, p. 1232).

9. Max Weber définit les notables comme "les personnes qui $1 /$ de par leur situation économique, sont en mesure, à titre secondaire, de diriger et d'administrer effectivement de façon continue un groupement quelconque, sans salaire ou contre un salaire nominal ou honorifique; 2 /jouissent d'une estime sociale - peu importe sur quoi celle-ci repose,- de sorte qu'ils ont la chance d'occuper des fonctions dans une démocratie directe formelle, en vertu de la confiance de ses membres, d'abord par acte volontaire, puis à la longue par tradition" (1971).

10. Dans une perspective récente, l'étude menée par Jean Joana sur les pratiques politiques des députés français au XIX siecle montre la dimension relative de cette distinction, tant les savoirs faire et les lieux de socialisation mis en avant sont proches (Joana, 1999).

11. Pierre Grémion propose une définition différente de celle des historiens du groupe des notables: "On peut discuter longuement pour énumérer les attributs des notables et fixer les contours de l'espèce. Mais ce débat essentialiste conduit à une impasse comme tous les débats de ce genre. Seule une définition fonctionnelle, liée à la position des individus concernés dans le système de rapports entre les structures territoriales de l'Etat et leur environnement, permet de progresser. Le notable dans cette perspective est un homme qui dispose d'un certain pouvoir pour agir sur l'appareil d'État à certains niveaux privilégiés et qui, par effet de retour, voit son pouvoir renforcé par le privilège que lui conferent ces contacts pour autant qu'ils soient sanctionnés par des résultats. Ainsi, le notable se définit moins par ces attributs personnels que par un système d'action constitué en étroite interdépendance avec les fonctions qu'assument les cellules territoriales de l'appareil administratif de l'État" (Grémion, 1976, p. 167).

12. Pour Dominique Lorrain "c'est avec la montée en puissance des villes, de nouveaux comportements sont apparus et un nouveau modele de l'action publique s'est fait jour. Les élus sont impliqués dans les dossiers. Ils ont adopté un comportement entrepreneurial qui combine la détermination des choix stratégiques avec une participation dans le suivi, afin de veiller aux dérapages et de corriger en temps réel. On passe d'une légitimité d'intercesseur à une légitimité par l'action. Les élus ne se contentent plus de rendre des services et d'administrer, ils agissent en propre", (Lorrain, 1993, p. 296).

13. Joseph Fontaine et Christian Le Bart soulignent la manière dont chez les chercheurs du CSO l'origine sociale et le fonctionnement même du notable ont prévalu sur la nature du mandat obtenu aux élections: "Pratiquant son mandat comme une activité secondaire, faiblement conditionné par les organisations politiques, le notable est surtout présenté comme un médiateur qui inscrit son action dans le cadre d'un 'jacobinisme apprivoise', fait d'interdépendance entre les administrations locales et les services de Etat" $(1994,13)$.

14. En précisant son objet d'étude, il souligne que les activités observées "ne sont cependant pas éclatées et aléatoires. Elles obéissent à un principe d'unité en ce sens qu'elles sont structurées, organisées et finalisées". Ainsi, selon Garraud, les analyses en termes de métier ciblent "le travail politique en tant que tel (...) les savoir-faire et les technologies nécessaires pour conquérir ou conserver le pouvoir politique" $(1994,34-35)$.

15. Pour Jean Louis Briquet, il est nécessaire d'analyser "les conditions effectives d'exercice d'un métier" ce qui l'amène "à privilégier une analyse en terme de rôle social, d'apprentissage des normes selon des normes considérées comme légitimes à l'intérieur de groupe professionnel" (1994, pp. 17-18).

16. "La mise en représentation n'est pas une dimension subalterne ou dérivée de l'action politique. $A$ l'inverse, on peut considérer qu'elle en constitue une condition fondamentale, commune à l'ensemble des sociétés humaines. De ces "liturgies politiques'(...) on peut discerner deux espèces: les unes exaltent l'unité, le consensus; les autres thêtralisent l'affrontement et la conflictualité. dans un pays comme la France, ces rituels scandent la vie politique, avec son alternance entre tensions et unanimisme" (Abelès, 1997, 251).

17. Pour Nobert Elias, "le terme de configuration sert à créer un outil conceptuel maniable, à l'aide duquel on peut desserrer la contrainte sociale qui nous oblige à penser et à parler comme si l'individu et la société étaient deux figures différentes et de surcroît antagonistes" (1991, pp. 156-157). À l'instar de ce sociologue, on considere qu'une configuration territoriale constitue une figure globale (correspondant à des situations concrètes d'echange social) où dominent des logiques d'interdépendance.

18. Nous retrouvons ici "la composante identitaire" de la médiation politique soulignée par Pierre Muller. Celleci "en constitue une dimension essentielle, dans la mesure où elle va fonder la vision qu'un groupe de sa 
propre place et de son rôle dans la société" (...) “Ce qui signifie qu'un acteur donné pourra se positionner comme médiateur à un moment donné et dans un contexte socio-organisationnel donné, mais ne pourra pas construire n'importe quel système de croyance en fonction de ses besoins stratégiques, les croyances étant le résultat d'une interaction très complexe entre la place du groupe dans la division du travail et l'identité construite à partir de cette place" (Muller, 1995, 162-163).

19. Le pouvoir local d'un l'élu se traduit par une fusion du rôle "d'entrepreneur territorial chargé d'argumenter publiquement la conversion d'atouts sectoriels en ressources pour le développement local" (Faure, 1997, p. 77).

20. On reprend ici les réflexions que nous avons développées au sein de l'équipe de chercheurs du CEPEL qui a suivi et analysé les élections du 15 mars 1998 en Languedoc-Roussillon. Les résultats de ces travaux sont publiés dans la revue Pôle Sud (cf. Alliès, alii., 1998).

21. La réflexion sur la formation d'un leadership écologiste dans le département de l'Hérault montre que pour imposer localement son pouvoir, la maîtrise de l'expertise environnementale est l'un des registres mobilisés (Genieys, Joana, 1996).

22. C'est ce que Burns appelle le rapport entre leaders and followers: "les leaders convainquent ceux qui les suivent (leurs followers) d'agir en faveur de certains objectifs qui représentent les valeurs et les motivations - les désirs et besoins, les aspirations et les attentes - aussi bien des leaders que des followers" $(1978,19)$.

23. A ce propos, Fontaine et Le Bart remarquent à juste titre qu'en concentrant l'analyse sur les activités de médiation de l'élu local, on se limite à "une perspective implicitement fonctionnaliste" occultant ainsi la dimension territoriale du pouvoir local $(1994,23)$.

24. Ici nous rejoignons les propos d'Aaron Wildavsky pour qui l'étude détaillée de l'action des leaders n'est véritablement pertinente que si on "replace ces acteurs dans des contextes ou des régimes politiques particuliers", $(1989,100)$.

25. L'analyse de la transition démocratique espagnole, nous a permis de montrer comment les leaders politiques de certaines périphéries, notamment la Catalogne, profite de la situation pour imposer une profonde reforme territoriale de l'État (Genieys, 1997, pp. 239-240).

26. Bien entendu, cette perspective n'est pas neuve mais fondée sur une longue tradition de recherche. Max Weber développe cette idée dans sa sociologie du pouvoir au sein des communautés politiques (1968, pp. 901-940). Il montre ainsi que dans ce type d'organisation sociale certains individus peuvent agir comme porteur d'un projet (au sens de träger) en vue de légitimer l'organisation politique du territoire. Ainsi, l'attachement à une conception sociale du territoire produit de la légitimité politique pour les leaders qui peuvent s'en revendiquer.

27. Il s'agit principalement des programmes LEADER (liaison entre actions de développement de l'économie rurale) mise en cuvre par l'Union Européenne au niveau infra-départemental à la fin des années 1980 , (Smith, 1995).

\section{Références}

Abéles Marc (1989), Jours tranquilles en 89, Paris, Odile Jacob.

Abélès Marc (1997), "La mise en représentation du politique", in M. Abélès, H.-P. Jeudy, (dir.), Anthropologie du politique, Paris, Armand Colin, pp. 247-271.

Allies Paul (1991), “Que sont nos notables devenus?”, Autrement, n 122, mai, pp. 108-119.

Allies Paul et alii. (1998), "Les élections régionales du 15 mars 1998 en Languedoc-Roussillon. Une nouvelle singularité politique régionale?", Pôle Sud n n 8 mai, pp. 5-41.

Bailey, Francis G. (1971) "Les règles du jeu politique" PUF Paris

Balme Richard et alii. (1994), Le territoire pour politiques: variations européennes, Paris, L'Harmattan.

Baraize François, Négrier Emmanuel (2000a), "Le projet de création d'une Communauté d'Agglomération à Montpellier", in GRALE-CNRS-CEP Ministère de l'Intérieur: Les premiers mois d'application de la loi du 12 juillet 1999, dactylo., pp. 266-284 (dir. G. Marcou)

Baraize, François \& Négrier Emmanuel (2000b) Communautés d'Agglomération et développement politique, communication au colloque européen "Décentralisation et Développement Durable", Reims 10-11 mai 2000, dactylo $12 \mathrm{p}$. 
Bayart Jean-François (1996), L'illusion identitaire, Paris, Fayard.

Birnbaum Pierre (1998), La France imaginée. Déclin des rêves unitaires?, Paris, Fayard.

Bourricaud François (1953), "La sociologie du leadership et son application à la théorie politique", Revue française de science politique, vol. 3. (3), pp. 445-470.

Briquet Jean-Louis (1994), "Communiquer en actes. Prescriptions de rôle et exercice quotidien du métier politique", Politix, $\mathrm{n}^{\circ} 28$, pp. 16-26.

Briquet Jean-Louis (1997), La tradition en mouvement, Paris, Belin.

Burns John MacGregor (1978), Leadership, New York, Harper and Row.

Charle Christophe (1997), "Légitimités en péril. Eléments pour une histoire comparée des élites et de l'État en France et en Europe occidentale (XXX'-XX' siecle)", Actes de la Recherche en Sciences Sociales, $\mathrm{n}^{\circ}$ 116/117 mars, pp. 39-52.

Chaussinand-Nogaret, Guy (dir.) (1991): "Histoire des élites en France du XVI' au XVII' siècle: I'honneur, le mérite, l'argent" Paris Tallandier

Cole Alistair, John Peter (1995), "Les réseaux locaux de politique publique en France et Grande-Bretagne”, in P. Le Gales, $M$. Thatcher, Les réseaux de politique publique, Paris, L'Harmattan, pp. 211-228.

Crozier, Michel - Thoenig Jean-Claude (1975): "La régulation des systèmes organisés complexes. Le cas du système de décision politico-administrative locale en France" Revue Française de Sociologie, Vol. XVI n 1, Janvier-Mars Paris

Dahl Robert (1961), Who governs?, New Haven, Yale University Press.

Duran Patrice, Thœnig Jean-Claude (1996), "L'Etat et la gestion publique territoriale", Revue franfaise de science politique, vol. 46 (4), pp. 580-623.

Elias Nobert (1991), Qu'est-ce que la sociologie?, La Tour d'Aigues, bd. de l'Aube.

Faure Alain (1992), Le village au politique, Paris, l'Harmattan.

Faure Alain (1997), "Les apprentissages du métier d'élu local. La tribu, le système, les arènes", Pôle Sud, n 7 novembre, pp. 72-80.

Faure Alain (mai 2000), "L'action publique locale entre territorialisation, territorialités et territoires. Pour une lecture politique des politiques locales", Lille, CLERSE, $20 \mathrm{p}$.

Faure Alain, Smith Andy (1998), "Espace rural, politiques publiques et cultures politiques", Ruralia, $\mathrm{n}^{\circ} 2$.

Fontaine Joseph, Le Bart Christian (dir.), (1994), Le métier de l'elu local Paris, l'Harmattan.

Freeman Ferero Thomas, Bloomberg Werner, Sunshine Morris, (1971), "Recherche de leaders dans les collectivités locales: comparaison de différentes approches", in P. Birnbaum, F. Chazel, Sociologie politique, Paris, PUF, pp. 234-247.

Friedberg Erhard (1993), Le pouvoir et la règle, Paris, Seuil.

Garraud Philippe (1989), Profession: homme politique, Paris, l'Harmattan.

Garraud Philippe (1994), "Le métier d'élu local: les contraintes d'un rôle", in J. Fontaine, C. Le Bart (dir.), Le métier d'elu local, Paris, L'Harmattan, pp. 29-54.

Gaudin Jean-Pierre (1995), "Politiques urbaines et négociations territoriales. Quelle légitimité pour les réseaux de politiques publiques?”, Revue Franfaise de Science Politique, vol. 45 (1), février, pp. 31-56.

Gaudin Jean-Pierre (1999), Gouverner par contrat, Paris, Presses de science po, 1999.

Gaxie Daniel (1973), Les professionnels de la politique, Paris, PUF.

Gaxie Daniel, Lehingue Patrick (1984), Enjeux municipaux, Paris, PUF.

Gaxie Daniel, (1997), Luttes d'institutions, Paris, L'Harmattan.

Genieys William, Joana Jean, (1996), “Un Janus notabiliaire: Yves Pietrasenta”, Sciences de la Societte, $\mathrm{n}^{\circ}$ 38, mai, pp. 143-158.

Genieys William (1997a). Les élites espagnoles face à l'Etat, Paris, L'Harmattan.

Genieys William (1997b), "Le territoire imaginaire du Pays Cathare", Pôle Sud, nº 7, novembre, pp. 118-131.

Genieys William (1997c), "Élites intermédiaires, frontières institutionnelles: Catalogne et Languedoc Roussillon", Revue Européenne des Migrations Internationales, vol. 13 (3), pp. 211 -227.

Genieys William, Smith Andy (1998), "Mobilisations politiques et modelles de leadership territorial. Analyse comparée d'une politique publique européenne et infra-régionale", in E. Négrier, B. Jouve (dir.), Que gouvernent les régionsd'Europe?, Paris, L'Harmattan, pp. 205-221. 
Genieys William (1999), "Les experts du développement local face à l'État. La genèse du Pays cathare”, in R. Balme, A. Faure, A. Mabileau, Les politiques locales en mouvement Paris, Presses de Science po.

Genieys William, Smith Andy (1999), Languedoc-Roussillon. Monographie régionale, Bruxelles (DG VI), Rapport de recherche PAC-REGIO, $60 \mathrm{p}$.

Grémion Pierre (1976), Le Pouvoir périphérique, Paris, Seuil.

Jessop Bop (1996), "A neo-Gramscian approach to the regulation of urban regimes: accumulation strategies, hegemonic projects and governance", in M. Lauria, (ed.), Reconstructing urban regime theory, London, Sage

Joana Jean (1999), Pratiques politiques des députés frangais au xixe siècle, Paris, L'Harmattan.

Jobert Bruno (1996), "Rhétorique politique, controverses scientifiques et construction des normes institutionnelles: esquisse d'un parcours de recherche", in A. Faure, G. Pollet, P. Warin, (dir.), La construction du sens dans les politiques publiques, Paris, L'Harmattan, pp. 13-23.

Lagroye Jacques (1973), Société et politique. Chaban Delmas, Paris, Pédone.

Le Bart Christian (1992), La rhétorique du maire entrepreneur, Paris, Pédone.

Le Gales Patrick (1995), "Du gouvernement des villes à la gouvernance urbaine", Revue française de science politique, vol. 45 (1), pp. 57-95.

Le Pape Yves, Smith Andy (1999), "Décentralisations et Agricultures: analyse comparée de deux régions françaises", Politique et management public, Vol. XVI n 4, décembre pp. 53-74

Lorrain Dominique (1993), "Après la décentralisation. L'action publique flexible”, Sociologie du Travaih $\mathrm{n}^{\circ} 3$, pp. 285-307.

Mabileau Albert, (1997), "Les génies invisibles du local. Faux-semblants et dynamiques de la décentralisation", Reure franfaise de science politique, vol. 47 (3-4) juin-2oût 1997, pp. 340-376.

Muller Pierre (1995), "Les politiques publiques comme construction d'un rapport au monde", in A. Faure, G. Pollet, P. Warin, (dir.), La construction du sens dans les politiques publiques Paris, L'Harmattan, pp. 153-179.

Négrier Emmanuel, (1998), "Une action publique sans coopération politique: le style languedocien de politique régionale" Pôle Sud n ${ }^{\circ} 8$, pp. 42-54

Paige Glenn, (1977), The scientific study of political leadership, New York, The Free Press.

Pôle Sud, Elites, politiques, et territoires, $\mathrm{n}^{\circ} 7$ novembre 1997,143 p.

Pourcher Yves (1987), Les maîtres de granit. Les notables de Lozère du XVIr à nos jours, Paris, Olivier Orban.

Rondin Jacques (1985), Le sacre des notables, Paris, Fayard.

Smith Andy (1995), L'Europe au miroir du local. Les fonds structurels et les zones rurales en France, en Espagne et au Royaume-Uni, Paris, l'Harmattan.

Smyrl, Marc (1995): "From regional policy to European networks: interregional divergence in the implementation of the EC Structural Funds in France" European University Institute, Firenze, Working Paper

Starratt Robert (1993), The drama of leadership, London, The Falmer Press.

Taylor Charles (1989), Sources of the self, London, Macmillan.

Tucker Robert (1995), Politics as leadership, Columbia, University of Missouri Press.

Tudesq André Jean (1964), Les Grands notables en France (1840-1849), Paris, PUF, 2 vol.

Weber Max (1968), Economy and Society. An outline of interpretative sociology, New York, The Free Press.

Weber Max (1971), Economie et societe, Paris, Plon.

Wildavsky Aaron (1989), "A cultural theory of leadership", in B. Jones (ed.), Leadership and politics, Laurence, University of Kansas press, pp. 87-113.

Worms Jean-Pierre (1966), "Le prefet et ses notables", Sociologie du travaih 3, pp. 249-275. 